\section{Successful Endoscopic \\ Removal of a Large Fibrovascular Polyp of the Esophagus}

F. Scintu, G. D’Alia, M. Cabras, M. Pisano, G. Casula

Dept. of General Surgery II, University of Cagliari,

Binaghi Hospital, Cagliari, Italy

\section{Corresponding Author}

Francesco Scintu, M.D.

Chirurgia Generale II

Universitá di Cagliari

Ospedale Binaghi

Via Is Guadazzonis 3

09126 Cagliari

Italy

Fax: + 39-70304462

E-mail: scintu@vaxca1.unica.it

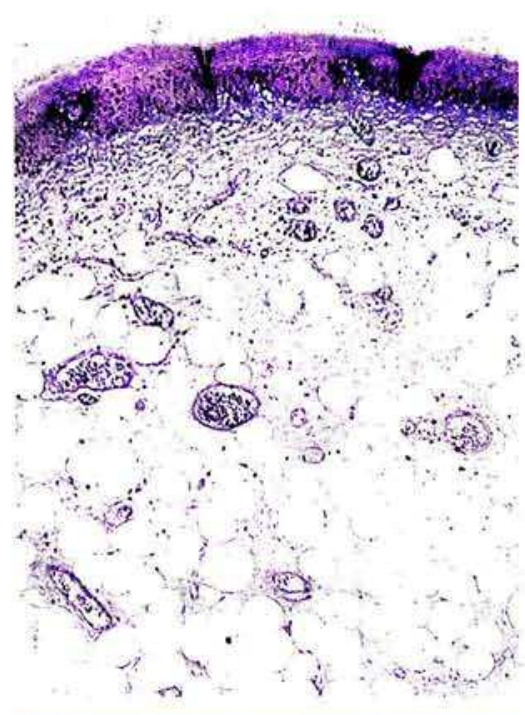

Fibrovascular polyps of the esophagus are rare, and composed of fibrovascular and adipose tissue (Figure 1). Larger polyps attached high in the cervical esophagus should be excised, because regurgitation can lead to asphyxia. Resection is the treatment of choice, as endoscopic polypectomy is inadvisable because of the danger of hemorrhage. In our experience, however, the latter can be accomplished uneventfully (Figure 2). Endoscopic removal can be proposed for polyps with narrow stalks, preferably under general anesthesia to protect the airways if there is bleeding.

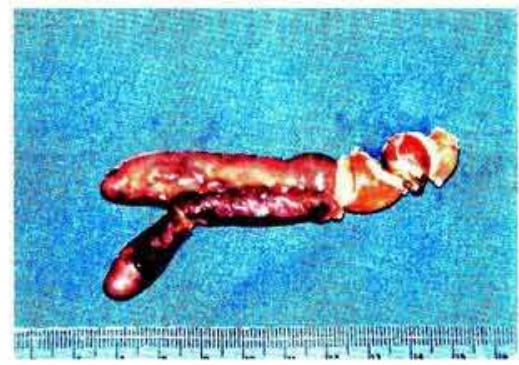

